\title{
Health Information Systems Supporting Health and Resiliency Through Improved Decision-making
}

\author{
David Ring ${ }^{1}$; William M. Tierney ${ }^{2}$ \\ ${ }^{1}$ Department of Surgery \& Perioperative Care, Dell Medical School, University of Texas at Austin, Austin, TX, USA; \\ 2Department of Population Health, Dell Medical School, University of Texas at Austin, Austin, TX, USA \\ Correspondence to: \\ William M. Tierney, MD \\ Professor and Chair, Department of Population Health \\ Dell Medical School, University of Texas at Austin \\ 1912 Speedway, Suite 562 \\ Austin, Texas 78712 \\ USA \\ E-mail: tierney@utexas.edu \\ Methods Inf Med 2017; 56(Open): e11-e12 \\ https://doi.org/10.3414/ME16-25-0001 \\ published: February 1, 2017
}

We wish all illness was like strep throat: immediate, accurate diagnosis and rapid cure. But illness is far more than pathophysiology. And the vast majority of pathophysiology can only be modified or palliated, not cured. Variations in resiliency account for the observation that a person can be very ill without objectively verifiable disease or healthy in spite of advanced pathology. Resiliency is optimized by greater resources, both personal and social. Personal factors that increase resiliency include meaning and purpose, better mood, less stress, more effective coping strategies, and greater knowledge and understanding. Social factors that increase resiliency include financial and relationship security; a strong network of family, friend, and community support; collective meaning and purpose; collective knowledge and awareness; and ready access to expertise and technology (a safety net). Effective self-care in the form of healthy habits and adherence to effective treatments also depends on resiliency. People must feel they are capable and worthy of good health in order to achieve it. And they must be hopeful of a better, healthy future to invest today in healthpromoting activities that cost money, time, and effort.

De Quiros and colleagues [1] are curious about the ways that health technology, specifically health information systems
(HIS) might 1) create a context that makes healthy behaviors more intuitive, appealing, and facile; and 2) address illness from the biopsychosocial framework [2], measuring and accounting for the psychological, sociocultural, and environmental factors that affect health. Technology can be used to create social constructs to optimize health. This framework can be interpreted as an attempt to increase resiliency through increasing self-efficacy and activation.

Technology can map health assets to form a scorecard of psychosocial opportunities and barriers for improved health. For example, in addition to screening for symptoms of depression and less effective coping skills, we can catalogue and study the impact of personal and social health resources. Research can determine which investments to enhance personal and social resources have the greatest impact on health for their relative cost in terms of money, time, and effort (i.e. the greatest value). Technology can provide data to inform program improvement and culture change endeavors. Technology can also inform modeling which can direct us to interventions most likely to have an impact.

Technology can also help people be more independent (e.g. seamless monitoring of blood pressure linked to algorithmic evidence-based, acceptable treatment modifications); encourage healthy habits; learn to value resiliency training and make it accessible and appealing; and see all their treatment options - getting past first impressions that might be based on misconceptions.

De Quiros and colleagues correctly note that current HIS applications don't capture and use data either on the social determinants of health or patient factors (e.g. preferences and beliefs) that affect their decision-making. Unfortunately, neither they nor any of those commenting on this article [3] offer concrete suggestions on how HIS might capture, store, and use these data. For example, nutrition advice given to a single mother with three children could be informed by knowing that she has little money, no automobile, and lives four blocks from the nearest bus station and four miles from the nearest grocery store selling fresh fruit and vegetables.

De Quiros et al. take the approach that the electronic health records (EHRs) should be constructed to capture the factors that affect patients' acceptance of clinicians' decisions. More useful, in our opinion, is shared decision-making where providers and patients engage in open and frank dialogue about patients' health status, its implications for quality and quantity of life, and health care and personal health options that might affect them. Patients are more likely to adhere to decisions they help make, and engaging patients in decisionmaking can be enhanced if patients have access to their EHRs and can store information directly into them. Who better than the patient and perhaps his or her family and caregivers to enter and update information on personal social and behavioral determinants of health? Instead of having different silos for provider and patient data, there are no technical barriers to creating 
and using EHRs in which both patients and providers can actively participate. Sadly, in the U.S. EHRs are primarily used to document and justify billing, and EHR vendors have little incentive to do the kinds of development necessary to support patient-provider sharing of information and decision-making. Perhaps the academic biomedical informatics community, which was instrumental in the earliest developing, testing, and demonstrating the value of EHRs, can embrace the mission of transforming them into a platform for effective provider-patient communication and decision-making [4]. Successful examples include OpenNotes [5], where patients have access to their health care providers' visit notes, and PeoplePower [6], an approach to shared decision-making embracing the notion of "nothing about me without me" that calls for information and communication technologies that allow patients and clinicians to contribute actively to the patient record, sharing transcripts of encounters, and moving patient education to their homes, schools, and community-based organizations.

We need to move to a new era of Precision Health where health care and people's lifestyles are supported by individualized and acceptable decisions and choices that are embraced by patients and enhance health and wellbeing. Both health care and caring for one's own health are information-intensive. Relevant information needs to be readily available to health care providers and their patients and their families and caregivers to enhance health and avoid the expensive, uncomfortable, and sometimes dangerous health conditions and their treatments. The onus is on us in the academic biomedical informatics community to meet this challenge and develop, implement, study, and disseminate the needed revolution in health information technology.

\section{References}

1. De Quiros FGB, Dawidowski AR, Figar S. Representation of people's decisions in health information systems: a complementary approach for understanding health care systems and population health. Methods Inf Med. 2017; 56(Open): e13-e19.

2. Engel GL. The clinical application of the biopsychosocial model. Am J Psychiatry. 1980; 137(5): 535-544.

3. Al-Shorbaji N, Borycki E, Kimura M, Lehmann CU, Lorenzi NM, Moura L, Winter A. Discussion of "Representation of People's Decisions in Health Information Systems: A Complementary Approach for Understanding Health Care Systems and Population Health". Methods Inf Med. 2017; 56(Open): e20-e29.

4. Payne TH, Corley S, Cullen TA, et al. Report of the AMIA EHR-2020 Task Force on the status and future direction of EHRs. J Am Med Inform Assoc. 2015; 22(5): 1102-1110.

5. Wolff JL, Darer JD, Berger A, et al. Inviting patients and care partners to read doctors' notes: OpenNotes and shared access to electronic medical records. J Am Med Inform Assoc. Forthcoming 2017.

6. Delbanco T, Berwick DM, Boufford JI, et al. Healthcare in a land called PeoplePower: nothing about me without me. Health Expect. 2001; 4(3): 144-150. 